\title{
Progression of coronary artery calcification in patients with type 1 diabetes mellitus with and without kidney disease
}

Patients with type 1 diabetes mellitus (T1DM) - even those without evidence of kidney disease - are at increased risk of coronary artery calcification (CAC) progression; however, the risk of CAC progression is amplified in patients with worsening renal function, according to new research. "Prevention of diabetic kidney disease is extremely important, but our study shows it is unlikely that prevention of diabetic kidney disease will completely eliminate the additional risk of cardiovascular disease in patients with T1DM," explains investigator David Maahs.

Diabetic nephropathy is an important risk factor for cardiovascular disease. To assess the association of diabetic kidney disease with accelerated atherosclerosis, Maahs and colleagues involved in the Coronary Artery Calcification in Type 1 Diabetes (CACTI) study investigated the ability of albumin-to-creatinine ratio (ACR) and estimated glomerular filtration 4

...even in the absence of kidney disease ... the risk of CAC progression remained increased in people with T1DM... 77

rate (eGFR) to predict CAC progression over 6 years of follow-up in patients with $(n=489)$ and without $(n=577)$ T1DM.

The researchers found that in patients with T1DM, increasing categories of baseline ACR $(<10 \mu \mathrm{g} / \mathrm{mg}, 10-30 \mu \mathrm{g} / \mathrm{mg}$, and $>30 \mu \mathrm{g} / \mathrm{mg}$ ) were associated with increases in the risk of CAC progression (ORs 2.15, 7.19, and 18.09, respectively), compared with the risk in nondiabetic individuals. Baseline eGFR $<60 \mathrm{ml} /$ $\mathrm{min} / 1.73 \mathrm{~m}^{2}$ as assessed using three CKD-EPI equations was also associated with an increased risk of CAC progression in patients with T1DM compared to the risk in those without (ORs 5.02-7.89 for the three equations). However, the researchers also noted that the odds for
CAC progression were also higher in patients with T1DM and eGFR $>60 \mathrm{ml} /$ $\mathrm{min} / 1.73 \mathrm{~m}^{2}$ than in nondiabetic patients. "Similar to the FinnDiane and the Pittsburgh Epidemiology of Diabetes Complications (EDC) studies, we found that cardiovascular disease risk increases with increasing evidence of kidney disease," explains Maahs. "One distinction with our data is that even in the absence of kidney disease, that is, with normal eGFR and ACR $<10 \mu \mathrm{g} / \mathrm{mg}$, the risk of CAC progression remained increased in people with T1DM compared with nondiabetics. These findings contrast with the FinnDiane and EDC studies, but are consistent with the recent findings of the Scottish registry linkage study."

Susan J. Allison

Original article Maahs, D. M. et al. Impaired renal function further increases odds of 6-year coronary artery calcification progression in adults with type 1 diabetes. Diabetes Care doi:10.2337/dc12-2538 